\title{
Self-Diffusion of Lithium in Amorphous Lithium Niobate Layers
}

\author{
By Johanna Rahn ${ }^{1, *}$, Erwin Hüger ${ }^{1}$, Lars Dörrer ${ }^{1}$, Benjamin Ruprecht ${ }^{2}$, \\ Paul Heitjans ${ }^{2,3}$, and Harald Schmidt ${ }^{1,3}$ \\ ${ }^{1}$ Institut für Metallurgie, Technische Universität Clausthal, Clausthal-Zellerfeld, Germany \\ ${ }^{2}$ Institut für Physikalische Chemie und Elektrochemie, Leibniz Universität Hannover, Germany \\ ${ }^{3}$ ZFM - Zentrum für Festkörperchemie und Neue Materialien, Hannover, Germany
}

(Received January 23, 2012; accepted in revised form March 8, 2012)

(Published online May 14, 2012)

\section{Amorphous Lithium Niobate / Self-Diffusion / Isotope Hetero-Structures / Secondary Ion Mass Spectrometry}

\begin{abstract}
We investigated lithium self-diffusion in amorphous lithium niobate layers between 298 and $423 \mathrm{~K}$. For the experiments, amorphous ${ }^{6} \mathrm{LiNbO}_{3} /{ }^{7} \mathrm{LiNbO}_{3}$ isotope hetero-structures were deposited by ion beam sputtering and analysed after diffusion annealing by secondary ion mass spectrometry (SIMS). This arrangement allows one to study pure isotope interdiffusion. The results show that the diffusivities obey the Arrhenius law with an activation enthalpy of $0.7 \mathrm{eV}$, which is considerably lower than the activation enthalpy found for $\mathrm{LiNbO}_{3}$ single crystals in literature. Consequently, the Li diffusivities are higher by at least eight orders of magnitude in the amorphous samples in the temperature range studied.
\end{abstract}

\section{Introduction}

Lithium niobate $\left(\mathrm{LiNbO}_{3}\right)$ in its single crystalline state is a very interesting technological oxide with extraordinary ferroelectric, piezoelectric, electro-optic and acoustic properties [1,2]. It does not exist as a stable compound in nature, however, it can be grown as a single crystal from the melt by the Czochralski method. Lithium niobate is stable over a wide solid solution range (44.0 to $50.5 \mathrm{~mol} \% \mathrm{Li}_{2} \mathrm{O}$ [3]). $\mathrm{A} \mathrm{LiNbO}_{3}$ single crystal is a relatively slow three-dimensional ion conductor $\left(\sim 10^{-12} \mathrm{~S} / \mathrm{cm}\right.$ at $500 \mathrm{~K}$ [4]) and consequently not suitable for technical applications in, e.g. solid electrolytes of secondary Li ion batteries [5]. However if structural disorder is introduced, e.g., by changing the structural state from single crystalline to amorphous, the ion mobility and therefore the conductivity is expected to increase significantly. Consequently, this material is an ideal model system to study how structural disorder may modify ionic diffusivity.

\footnotetext{
* Corresponding author. E-mail: johanna.rahn@tu-clausthal.de
} 


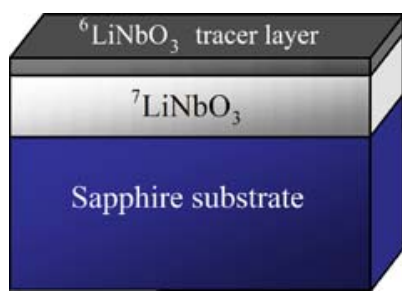

Fig. 1. Sketch of the isotope hetero-structure on a sapphire substrate after the sputtering process. Thicknesses: ${ }^{6} \mathrm{LiNbO}_{3}$ layer $\sim 20-50 \mathrm{~nm} ;{ }^{7} \mathrm{LiNbO}_{3}$ layer $\sim 800 \mathrm{~nm}$.

Most of $\mathrm{Li}$ diffusion experiments in $\mathrm{LiNbO}_{3}$ have been conducted by nuclear magnetic resonance spectroscopy (NMR) [4,6-10] and impedance spectroscopy studies $[11,12]$. The only studies on Li diffusion based on mass tracers are limited to single crystalline $\mathrm{LiNbO}_{3}$ [13-15]. Consequently, in the present study Li self-diffusion studies are carried out on amorphous lithium niobate at temperatures between 298 and $423 \mathrm{~K}$ using secondary ion mass spectrometry. The results are compared to studies on Li selfdiffusion in single crystals as given in literature [15].

In order to realize tracer diffusion studies for Li containing solids, stable tracers have to be used. Radioactive tracer isotopes with a half-life larger than some seconds for radio-tracer measurements do not exist. Lithium has two stable isotopes ${ }^{6} \mathrm{Li}(7.5 \%)$ and ${ }^{7} \mathrm{Li}(92.5 \%)$. In the present study we used ${ }^{6} \mathrm{LiNbO}_{3} /{ }^{7} \mathrm{LiNbO}_{3}$ isotope hetero-structures for analysis. This is an extremely reliable method to study self-diffusion processes in solids because pure isotope interdiffusion takes place undisturbed by chemical gradients [16-20].

\section{Experimental}

For tracer diffusion studies in amorphous lithium niobate we prepared so-called isotope hetero-structures (Fig. 1). As substrate we used a $10 \times 10 \times 0.5 \mathrm{~mm}^{3} c$-axis oriented polished sapphire single crystal, supplied by CrysTec (Berlin, Germany). First, an amorphous layer of ${ }^{7} \mathrm{LiNbO}_{3}$ of $800 \mathrm{~nm}$ thickness was deposited. Afterwards, the tracer layer of ${ }^{6} \mathrm{LiNbO}_{3}(\sim 20-50 \mathrm{~nm})$ was deposited (Fig. 1). Each isotope layer was prepared by ion beam sputtering using a commercial set-up (IBC 681, Gatan) equipped with two penning ion sources. The deposition was done at $5 \mathrm{keV}$ and a current of about $200 \mu \mathrm{A}$ in Argon at an operation pressure of $5 \times 10^{-5}$ mbar. The base pressure was better than $5 \times 10^{-7}$ mbar. The sputter process was carried out at room temperature and no significant heating of the substrate took place. Sharp material interfaces can be produced. The structural state of the deposited $\mathrm{LiNbO}_{3}$ was characterized using grazing incidence X-ray diffractometry on a Bruker D5000 diffractometer.

Sputter targets were prepared by solid state syntheses. In an agate mortar coarse $\mathrm{Nb}_{2} \mathrm{O}_{5}(99.9985 \%$, Alfa Aesar) was pestled to a fine powder and mixed with enriched ${ }^{6} \mathrm{Li}_{2} \mathrm{CO}_{3}\left(96 \%{ }^{6} \mathrm{Li}\right.$, Eurisotop) or ${ }^{7} \mathrm{Li}_{2} \mathrm{CO}_{3}\left(99.9 \%{ }^{7} \mathrm{Li}\right.$, Alfa Aesar) respectively. After subsequent ball milling the powder mixture in a SPEX $8000 \mathrm{M}$ shaker mill, pellets of 


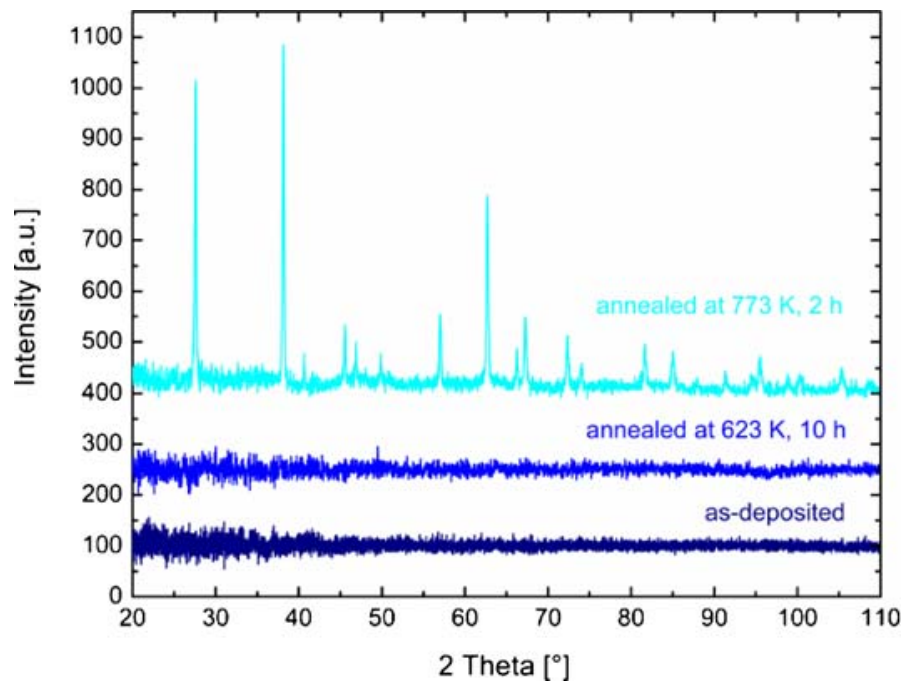

Fig. 2. X-ray diffraction patterns of a $\mathrm{LiNbO}_{3}$ sputter layer after deposition and after annealing at $623 \mathrm{~K}$ and $773 \mathrm{~K}$, respectively. All Bragg peaks correspond to the $\mathrm{LiNbO}_{3}$ phase (space group R3c) [1]. For clarity, the patterns are shifted to higher intensity.

$2 \mathrm{~cm}$ in diameter were pressed and heated to $973 \mathrm{~K}$ with a rate of $2 \mathrm{~K} / \mathrm{min}$. The reaction step was followed by a sintering process at $1173 \mathrm{~K}$ for $12 \mathrm{~h}$, which yielded polycrystalline dense targets. In order to account for loss of lithium in the sputtering process, the molar ratio of oxide and carbonate was chosen as $5: 6$. This results in targets of the composition $90 \% \mathrm{LiNbO}_{3}+10 \% \mathrm{Li}_{3} \mathrm{NbO}_{4}$.

For the $\mathrm{Li}$ isotope interdiffusion experiments the prepared samples were annealed in an Argon atmosphere between 333 and $423 \mathrm{~K}$ using a commercial rapid annealing setup (AO 500, MBE, Germany). The annealing time was between $10 \mathrm{sec}$ and $2 \mathrm{~min}$.

Secondary ion mass spectrometry (SIMS) investigations were carried out using a CAMECA IMS-3F machine. Due to electrical charging during the measurements we used an $\mathrm{O}^{-}$primary ion beam $(15 \mathrm{keV}, 30 \mathrm{nA})$. The sputtering area was of $250 \times$ $250 \mathrm{\mu m}^{2}$. However, for analysis of the isotopes in a double focused mass spectrometer the signal resulting from an area of about $60 \mu \mathrm{m} \times 60 \mu \mathrm{m}$ in the centre of the sputtering area is used in order to exclude crater edge effects.

The secondary ion intensities of ${ }^{6} \mathrm{Li}^{+}$and ${ }^{7} \mathrm{Li}^{+}$ions were recorded as a function of sputter time using the depth profiling mode. Since the two Li isotopes are chemically identical (neglecting the small isotope effect), for diffusion analysis the intensity of the signals is converted into ${ }^{6} \mathrm{Li}$ atomic fractions $c(x, t)$ according to

$$
c(x, t)=\frac{I\left({ }^{6} \mathrm{Li}\right)}{I\left({ }^{6} \mathrm{Li}\right)+I\left({ }^{7} \mathrm{Li}\right)}
$$

The depth of each measurement crater was quantified by a mechanical profilometer (Tencor, Alphastep). 


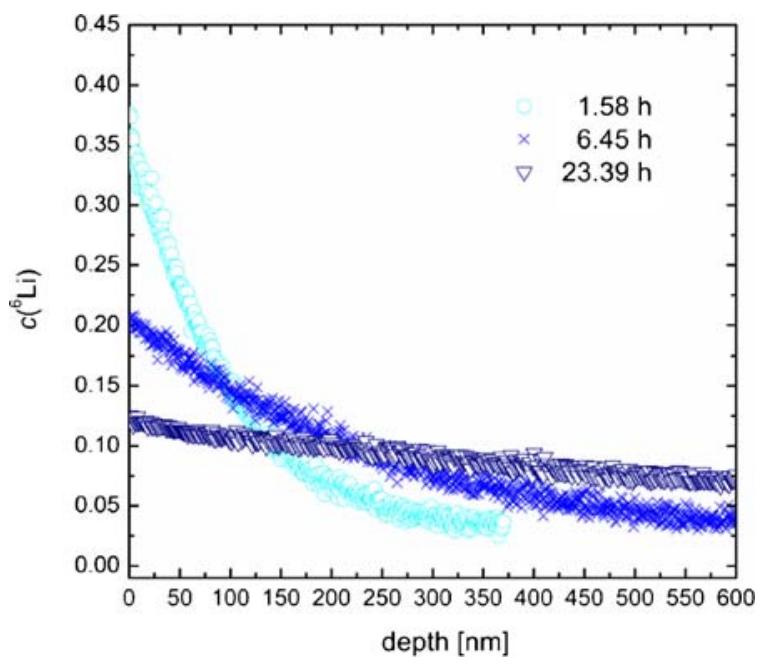

Fig. 3. Atomic fraction of ${ }^{6} \mathrm{Li}$ as a function of depth, measured with SIMS for different storing times at room temperature without any additional annealing.

\section{Results}

In order to investigate the structural state of the samples, X-ray diffraction patterns of conventional $300 \mathrm{~nm}$ thick $\mathrm{LiNbO}_{3}$ layers in the as-deposited state and after annealing were recorded, which are given in Fig. 2.

As obvious, the layers are X-ray amorphous after deposition. Annealing up to $623 \mathrm{~K}$ for $10 \mathrm{~h}$ showed that still no crystallization occurs. Consequently, the diffusion experiments were all done on amorphous samples. Investigations on sputter layers annealed at $773 \mathrm{~K}$ exhibited polycrystalline phase formation. Phase analysis was carried out using the program Powdercell for Windows 2.3. All Bragg peaks correspond to single phase $\mathrm{LiNbO}_{3}$ [1], while no secondary phase as, e.g., $\mathrm{Li}_{3} \mathrm{NbO}_{4}$ or $\mathrm{LiNb}_{3} \mathrm{O}_{8}$ has been found. This was checked for sputter layers of each target material. The excess $\mathrm{Li}_{2} \mathrm{O}$ present in the targets is lost during the sputtering process, as expected.

In Fig. 3 typical SIMS isotope depths profiles of as-deposited ${ }^{6} \mathrm{LiNbO}_{3} /{ }^{7} \mathrm{LiNbO}_{3}$ isotope hetero-structures are shown, which were stored in air at room temperature $(298 \mathrm{~K})$ for different times after deposition. The ${ }^{6} \mathrm{Li}$ fraction, $c(x, t)$, was plotted as a function of depth for various times. With increasing storage time the ${ }^{6} \mathrm{Li}$ successively penetrates into the ${ }^{7} \mathrm{LiNbO}_{3}$ film.

The results in Fig. 3 show that self-diffusion of ${ }^{6} \mathrm{Li}$ in the amorphous ${ }^{7} \mathrm{LiNbO}_{3}$ layer is fast, also at room temperature. After about $24 \mathrm{~h}$ of storing the penetration depth reached more than $800 \mathrm{~nm}$. This result implies that non negligible Li self-diffusion takes place also during sputtering and SIMS analysis. This has to be taken into account in the following analysis. The experimentally determined depth profiles was described by the following solution of Fick's second law for self-diffusion across an interface [21]

$$
c(x, t)=c_{\infty}+\frac{\left(c_{0}-c_{\infty}\right)}{2}\left[\operatorname{erf}\left(\frac{h+x}{R}\right)+\operatorname{erf}\left(\frac{h-x}{R}\right)\right]
$$




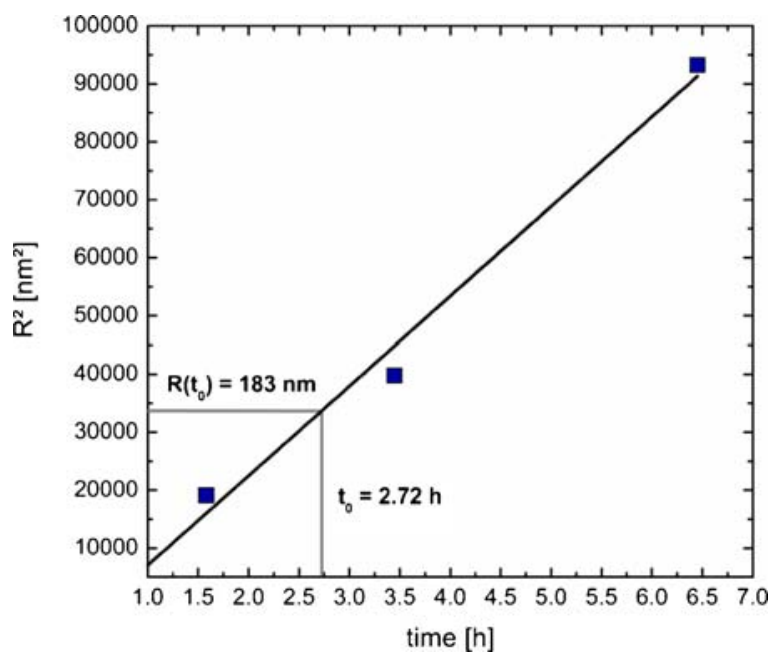

Fig. 4. $R^{2}$ as function of storage time at room temperature. As an example, the determination of $R\left(t_{0}\right)$ is illustrated for the sample annealed at $423 \mathrm{~K}\left(t_{0}=2.72 \mathrm{~h}, R\left(t_{0}\right)=183 \mathrm{~nm}\right)$. Experimental data: squares; linear fit: straight line.

where $c_{\infty}$ is the measured natural abundance of ${ }^{6} \mathrm{Li}$ in the amorphous ${ }^{7} \mathrm{LiNbO}_{3}$ layer and $c_{0}$ that in the ${ }^{6} \mathrm{LiNbO}_{3}$ tracer layer. The original thickness of the as-deposited tracer layer is denoted as $h$, which ranges between 20 and $50 \mathrm{~nm}$ for different samples. The quantity $R$ describes the broadening of the tracer profile. The self-diffusivity $D$ at time $t$ is determined from the difference in $R$ squared of the diffusion profile and of a starting profile, $R\left(t_{0}\right)$, according to

$$
D=\frac{\left(R^{2}-R^{2}\left(t_{0}\right)\right)}{4 t_{\mathrm{a}}}
$$

where $t_{\mathrm{a}}$ is the annealing time. In order to illustrate the calculation of $D$, a classical experiment is described in more detail. After deposition of the ${ }^{6} \mathrm{LiNbO}_{3}$ layer the sample was instantaneously transferred to the furnace and annealing was started. This time is termed $t_{\mathrm{s} 1}$, where diffusion takes place at room temperature. The annealing time at elevated temperature with accelerated diffusion is termed $t_{\mathrm{a}}$. Afterwards the cooled down sample is transferred to the SIMS machine during time $t_{\mathrm{s} 2}$. The time of the SIMS measurement at room temperature is termed $t_{\mathrm{SIMS}}$. Now $t_{0}$ is defined as $t_{0}=t_{\mathrm{s} 1}+t_{\mathrm{s} 2}+0.5 t_{\mathrm{SIMS}}$. The use of the factor 0.5 is a reliable approximation, which results in a negligible error in diffusivity. The quantities $t_{0}$ and $t_{\mathrm{a}}$ are fixed by the individual condition of each single experiment. Now, $R\left(t_{0}\right)$ is calculated from the experimental results done exclusively at room temperature as given in Fig. 4. It is shown that $R^{2}$ as a function of storage time at room temperature can be described by a straight line. The $R$ value corresponding to a given time $t_{0}$ is extracted from Fig. 4 . The procedure is illustrated for the sample annealed at $423 \mathrm{~K}$. For example, for $t_{0}=2.72 \mathrm{~h}$ we get $R\left(t_{0}\right)=183 \mathrm{~nm}$.

In Table 1 the results of two samples stored exclusively at room temperature are given. Here, $t_{\mathrm{a}}$ corresponds also to room temperature and is the time the sample is 
Table 1. Compilation of parameters of the diffusion experiments on as-deposited samples stored at room temperature; $t_{0}$ is the starting time, $t_{\mathrm{a}}$ is the annealing time, $R\left(t_{0}\right)$ is the diffusion length at $t_{0}$ as obtained from Fig. 4, and $R$ is the diffusion length obtained from the fit with Eq. (2). $D$ is the calculated lithium self-diffusivity. Typical relative errors attributed to the diffusivities from the fitting of Eq. (2) are about $30-40 \%$.

\begin{tabular}{lcccccc}
\hline Sample & $T[\mathrm{~K}]$ & $t_{0}[\mathrm{~h}]$ & $R\left(t_{0}\right)[\mathrm{nm}]$ & $t_{\mathrm{a}}[\mathrm{h}]$ & $R[\mathrm{~nm}]$ & $D\left[\mathrm{~m}^{2} / \mathrm{s}\right]$ \\
\hline 1 & 298 & 1.58 & 138 & 1.87 & 199 & $7.7 \times 10^{-19}$ \\
1 & 298 & 1.58 & 138 & 4.87 & 305 & $1.1 \times 10^{-18}$ \\
2 & 298 & 1.70 & 123 & 2.79 & 199 & $6.1 \times 10^{-19}$ \\
\hline
\end{tabular}

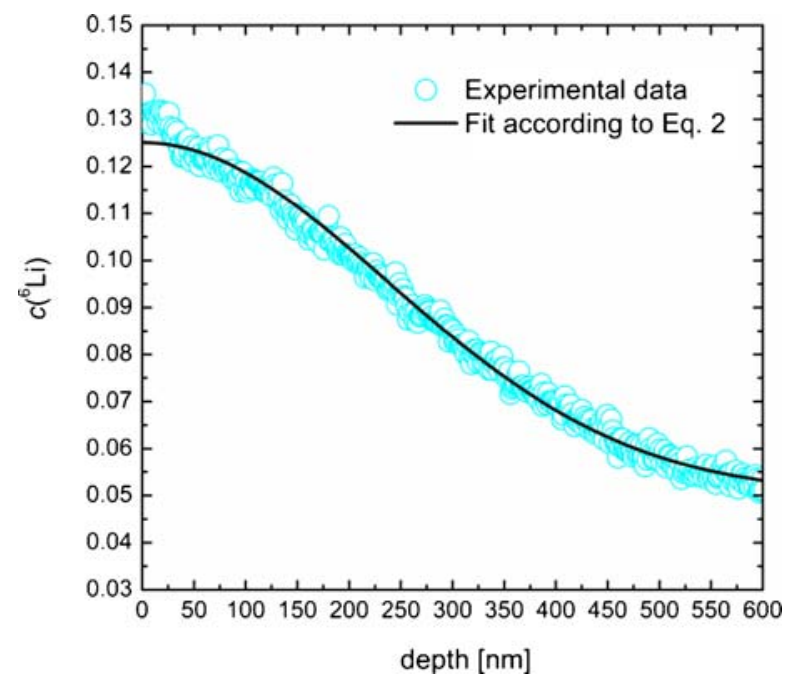

Fig. 5. Atomic fraction of ${ }^{6} \mathrm{Li}$ as a function of depth after annealing at $383 \mathrm{~K}$ for $2 \mathrm{~min}$. A least-squares fit of Eq. (2) to the experimental data is also shown ( $h=24 \mathrm{~nm}, R=327 \mathrm{~nm})$.

stored at room temperature between successive SIMS experiments. Based on the data of Table 1 corresponding diffusivities at room temperature are obtained. For samples annealed at elevated temperature the annealing time is used for $t_{\mathrm{a}}$.

In Fig. 5 an example for a typical experimentally determined isotope depth profile and the corresponding fit is illustrated $\left(T=383 \mathrm{~K}, t_{\mathrm{a}}=2 \mathrm{~min}\right)$. From this fit a value of $R=327 \mathrm{~nm}$ is derived. Further results obtained for different samples are given in Table 2 together with the calculated diffusivities. We have to note that not all depth profiles can be perfectly fitted by Eq. (2) at present, especially for low penetration depth $<50 \mathrm{~nm}$. This fact will be elucidated in closer detail in future.

From the room-temperature investigations of the as-deposited sample three diffusivities are obtained (Table 1). These are identical within error limits. Consequently, a possible annealing time dependence of diffusivities could not be confirmed in this study. All diffusivities are plotted in Fig. 6 as a function of reciprocal temperature. They 
Table 2. Compilation of parameters of the diffusion experiments at temperatures between 333 and $423 \mathrm{~K}$ done on different samples. $T$ is the annealing temperature, $t_{0}$ is the starting time, $t_{\mathrm{a}}$ is the annealing time, $R\left(t_{0}\right)$ is the diffusion length at $t_{0}$ as obtained from Fig. 4, and $R$ is the diffusion length obtained from the fit with Eq. (2). $D$ is the calculated lithium self-diffusivity. Typical relative errors attributed to the diffusivities from the fitting of Eq. (2) are about $30-40 \%$.

\begin{tabular}{lccccc}
\hline$T[\mathrm{~K}]$ & $t_{0}[\mathrm{~h}]$ & $R\left(t_{0}\right)[\mathrm{nm}]$ & $t_{\mathrm{a}}[\mathrm{s}]$ & $R[\mathrm{~nm}]$ & $D\left[\mathrm{~m}^{2} / \mathrm{s}\right]$ \\
\hline 333 & 1.12 & 101 & 120 & 132 & $1.5 \times 10^{-17}$ \\
343 & 1.59 & 107 & 120 & 166 & $3.3 \times 10^{-17}$ \\
353 & 1.81 & 127 & 120 & 269 & $1.2 \times 10^{-16}$ \\
363 & 1.54 & 101 & 120 & 264 & $1.2 \times 10^{-16}$ \\
373 & 1.47 & 115 & 60 & 277 & $2.6 \times 10^{-16}$ \\
383 & 1.51 & 100 & 120 & 327 & $2.0 \times 10^{-16}$ \\
423 & 2.72 & 183 & 10 & 347 & $2.2 \times 10^{-15}$ \\
\hline
\end{tabular}

\section{$\mathrm{T}\left[{ }^{\circ} \mathrm{C}\right]$}

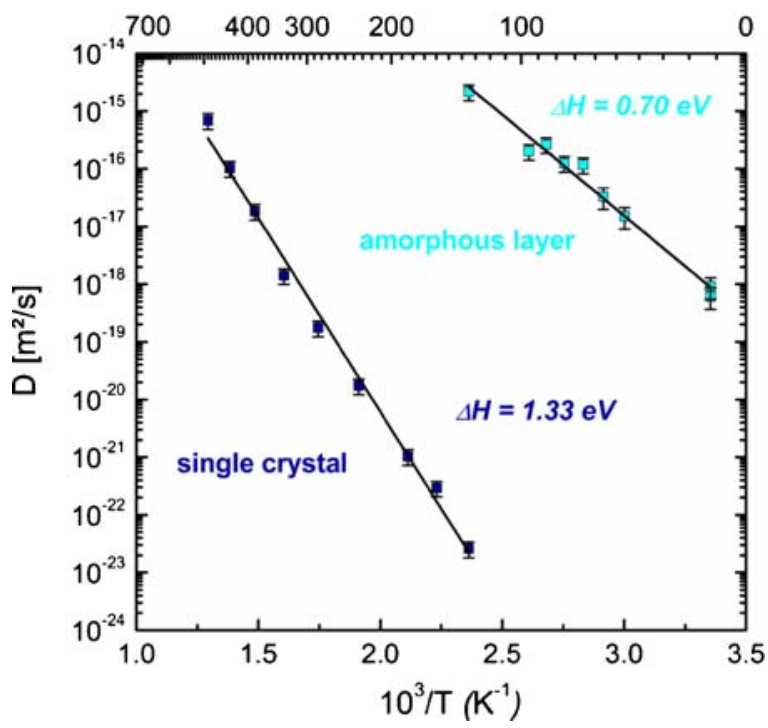

Fig. 6. Diffusivities $(D)$ of lithium in amorphous lithium niobate as a function of reciprocal temperature in comparison to the diffusivities of a lithium niobate single crystal [15].

obey the Arrhenius law

$$
D=D_{0} \exp \left(\frac{-\Delta H}{k_{\mathrm{B}} T}\right)
$$

where $k_{\mathrm{B}}$ is the Boltzmann constant and $T$ is the temperature. A least-squares fit of Eq. (4) to the diffusivities results in an activation enthalpy of $\Delta H=(0.70 \pm 0.03) \mathrm{eV}$ and a pre-exponential factor of $D_{0}=5.0 \times 10^{-7} \mathrm{~m}^{2} / \mathrm{s}$ (error: $\ln D_{0} / \mathrm{m}^{2} / \mathrm{s}= \pm 1.2$ ) in the temperature range between 298 and $423 \mathrm{~K}$. 


\section{Discussion}

In the following discussion the present results on lithium diffusion in amorphous lithium niobate are compared to those of a lithium niobate single crystal, as given in Ref. [15]. These diffusivities are also shown in Fig. 6. As obvious, the diffusivities in amorphous lithium niobate are higher by at least eight orders of magnitude in the temperature range investigated. This is a tremendous difference not commonly found for other types of ceramics [17]. It was further found in literature that diffusion in single crystals is mediated by single positively charged vacancies [15]. The activation enthalpy of self-diffusion is $1.33 \mathrm{eV}$. It consists of the vacancy migration enthalpy only. Structural vacancies are present whose concentration is fixed by a frozen-in defect structure formed during growth of the single crystal [15]. The activation enthalpy of diffusion in amorphous lithium niobate of $0.7 \mathrm{eV}$ is significantly lower by a factor of almost two. Further, it is proven that the pre-exponential factor of single crystalline $\mathrm{LiNbO}_{3}$ of $1.7 \times$ $10^{-7} \mathrm{~m}^{2} / \mathrm{s}$ (error: $\ln D_{0} / \mathrm{m}^{2} / \mathrm{s}= \pm 0.8$ ) and of amorphous $\mathrm{LiNbO}_{3}$ of $5.0 \times 10^{-7} \mathrm{~m}^{2} / \mathrm{s}$ (error: $\ln D_{0} / \mathrm{m}^{2} / \mathrm{s}= \pm 1.2$ ) are identical within error limits. This is a strong hint that despite of the different activation enthalpies a similar diffusion mechanism is present in both lithium niobate modifications. The pre-exponential factor is given by

$$
D_{0}=x a^{2} v_{0} \exp \left(\frac{\Delta S}{k_{\mathrm{B}}}\right)
$$

where $a$ is the Li-Li jump distance, $x$ is the mole fraction of (structural) lithium vacancies, $v_{0}$ is a characteristic vibrations frequency and $\Delta S$ is the entropy of ionic migration. A small modification of the Li-Li jump distance of $a=3.771 \AA$ [22] in amorphous $\mathrm{LiNbO}_{3}$ has no significant influence on the pre-exponential factor. The same is true for the vibration frequency of $v_{0}=2.5 \times 10^{13} / \mathrm{s}$. The migration entropy of vacancies in the single crystal is assumed to be $\Delta S \approx 0$ [15] and the mole fraction of lithium vacancies is given by $x=0.04$ [23]. Consequently, the identical pre-exponential factors mean that the latter two quantities $(\Delta S$ and $x$ ) are similar in amorphous and crystalline lithium niobate. We conclude that very likely in the amorphous modification diffusion takes place via vacancy-like defects with a similar vacancy concentration as in single crystals. This means that in the amorphous configuration localized defects are present, e.g. "point defects", where an atom is absent in the local short range order of the amorphous structure. If in contrast de-localized defects are present, like e.g. described by the free volume concept of metallic glasses [27], a significant higher entropy factor and also a higher pre-exponential factor would be expected. The same might be true for a possible collective diffusion mechanism. Considering the fact that in amorphous and crystalline lithium niobate a similar diffusion mechanism is present, the activation enthalpy attributed to the amorphous structure of $0.7 \mathrm{eV}$ is relatively low. The lower activation enthalpy corresponds to the much higher lithium mobility (at least eight orders). A qualitative explanation might be that in the amorphous state the atomic distances are slightly larger than in the crystalline state in average. Consequently, a lower migration enthalpy is necesssary for ionic movement.

In literature, no tracer diffusion studies on amorphous lithium niobate can be found. However, dc conductivities, $\sigma$, as determined by impedance spectroscopy, were published for sol-gel derived amorphous $\mathrm{LiNbO}_{3}$ between 320 and $450 \mathrm{~K}$ [4]. It was found 
that the quantity $\sigma T$ follows the Arrhenius law with an activation enthalpy of $0.59 \mathrm{eV}$. According to the Nernst-Einstein relation

$$
D=\frac{k_{\mathrm{B}} T \sigma}{n q^{2}}
$$

the diffusivity, $D$, is proportional to $\sigma T$, where $n$ denotes the number density of the conducting species, $q=e$ the electrical charge, $k_{\mathrm{B}}$ the Boltzmann constant, and $T$ the temperature. Consequently, the activation enthalpy of the present tracer diffusion study and of the conductivity study can be compared. The activation enthalpy found for the sol-gel derived material is lower by $20 \%$. This is not very surprising if materials are compared which were synthesized via completely different routes (sol-gel, ion beam sputtering) and which were also characterized by different methods (conductivity, mass tracer diffusion). Nevertheless, the extraordinarily high diffusivity found for the amorphous state is showed up in both studies.

\section{Conclusion}

Tracer diffusion experiments based on SIMS depth profiling have been carried out in order to investigate lithium self-diffusion in amorphous lithium niobate layers. We determined diffusivities in the temperature range between 298 and $423 \mathrm{~K}$. An Arrhenius behaviour is found with an activation enthalpy of $0.7 \mathrm{eV}$. In comparison to single crystalline $\mathrm{LiNbO}_{3}$ the pre-exponential factor is identical within error limits, while the activation enthalpy is lower by a factor of roughly two. This points to a similar diffusion mechanism via vacancy-like defects, which, however, takes place in the loosely packed amorphous structure. The overall diffusivities are several orders of magnitude higher than in the single crystal.

\section{Acknowledgement}

Financial support from the Deutsche Forschungsgemeinschaft (DFG) in the framework of the research unit FOR 1277 ("molife") is gratefully acknowledged.

\section{References}

1. T. Volk and M. Wöhlecke, Lithium Niobate, Springer, Berlin (2010).

2. K. K. Wong, Properties of Lithium Niobate, Inspec, London (2002).

3. P. Lerner, C. Legras, and J. Dumas, J. Cryst. Growth 3 (1968) 231.

4. P. Heitjans, M. Masoud, A. Feldhoff, and M. Wilkening, Faraday Discuss. 134 (2007) 67.

5. P. Knauth, Solid State Ionics 180 (2009) 911.

6. M. Wilkening, D. Bork, S. Indris, and P. Heitjans, Phys. Chem. Chem. Phys. 4 (2002) 3246.

7. M. Wilkening and P. Heitjans, Solid State Ionics 177 (2006) 3031.

8. T. K. Halstead, J. Chem. Phys. 53 (1970) 3427.

9. D. Bork and P. Heitjans, J. Phys. Chem. B 102 (1998) 7303.

10. D. Bork and P. Heitjans, P. J. Phys. Chem. B 105 (2001) 9162.

11. A. Mehta, E. K. Chang, and D. M. Smyth, J. Mater. Res. 6 (1991) 851.

12. M. Masoud and P. Heitjans, Def. Diff. Forum 237-240 (2005) 1016. 
13. D. P. Birnie III, J. Mater. Sci. 28 (1993) 302.

14. V. B. Ptashnik and T. Y. Dunaeva, Inorg. Mater. 21 (1985) 1814.

15. J. Rahn, E. Hüger, L. Dörrer, B. Ruprecht, P. Heitjans, and H. Schmidt, Phys. Chem. Chem. Phys. 14 (2012) 2427.

16. H. Schmidt, G. Borchardt, C. Schmalzried, R. Telle, S. Weber, and H. Scherrer, J. Appl. Phys. 93 (2003) 907.

17. H. Schmidt, U. Geckle, and M. Bruns, Phys. Rev. B. 74 (2006) 045203.

18. E. Hüger, U. Tietze, D. Lott, H. Bracht, E. E. Haller, D. Bougeard, and H. Schmidt, Appl. Phys. Lett. 93 (2008) 162104.

19. H. D. Fuchs, W. Walukiewicz, E. E. Haller, W. Dondl, R. Schorer, G. Abstreiter, A. I. Rudnev, and A. V. Tikhomirov, Phys. Rev. B 51 (1995) 16817.

20. H. A. Bracht, H. H. Silvestri, and E.E. Haller, Solid State Commun. 133 (2005) 727.

21. J. Crank, The Mathematics of Diffusion, Oxford University Press, Oxford (1975).

22. H. Xu, D. Lee, S. B. Sinnott, V. Dierolf, V. Gopalan, and S. R. Phillpot, J. Phys.: Condens. Matter 22 (2010) 135002.

23. J. Shi, H. Fritze, G. Borchardt, and K.-D. Becker, Phys. Chem. Chem. Phys. 13 (2011) 6925.

24. P. J. Jorgensen and R. W. Bartlett, J. Phys. Chem. Solids 30 (1969) 2639.

25. P. Fielitz, G. Borchardt, R. De Souza, M. Martin, M. Masoud, and P. Heitjans, Sol. State Sci. 10 (2008) 746.

26. P. Fielitz, O. Schneider, G. Borchardt, A. Weidenfelder, H. Fritze, J. Shi, K. D. Becker, S. Ganschow, and B. Bertram, Solid State Ionics 189 (2011) 1.

27. F. Faupel, W. Frank, M.-P. Macht, H. Mehrer, V. Naundorf, K. Rätzke, H. R. Schober, S. K. Sharma, and H. Teichler, Rev. Mod. Phys. 75 (2003) 236. 